\author{
Апарина О.П. ${ }^{1}$, Стукалова О.В. ${ }^{1 *}$, Терновой С.К. ${ }^{1,2}$ \\ ${ }^{1}$ НИИ клинической кардиологии им. А.Л. Мясникова ФГБУ “Российский кардиологический научно-производственный \\ комплекс" Минздрава России, Москва, Россия \\ 2 ФГАОУ ВО Первый Московский государственный медицинский университет им. И.М. Сеченова Минздрава России \\ (Сеченовский Университет), Москва, Россия
}

\title{
Magnetic Resonance Imaging with Delayed Contrast Enhancement in Atrial Fibrillation: First Advances and Perspectives
}

\author{
Aparina O.P. ${ }^{1}$, Stukalova O.V. ${ }^{1 *}$, Ternovoy S.K. ${ }^{1,2}$ \\ ${ }^{1}$ A.L. Mjasnikov Institute of the clinical cardiology Russian Cardiologic research and production complex, Moscow, Russia \\ 2 I.M. Sechenov First Moscow state medical university (Sechenov University), Moscow, Russia
}

В статье рассмотрены возможности неинвазивной оценки фиброза левого предсердия при фибрилляции предсердий при помощи магнитно-резонансной томографии (МРТ) с отсроченным контрастированием и первый опыт применения этой технологии в клинической практике.

В ряде проспективных исследований показано, что выраженность поражения предсердного миокарда может влиять на клиническое течение аритмии и результаты ее лечения. Ряд отечественных и европейских экспертов-морфологов и специалистов МРТ в совместном консенсусе предлагают ввести термин "фиброзная кардиомиопатия предсердий”, отражающий структурно-функциональную патологию предсердий, имеющую клиническую значимость при фибрилляции предсердий. Эти данные обосновывают потребность в неинвазивном обследовании широкого круга кардиологических пациентов с целью оценки структуры предсердного миокарда.

Совершенствование технологии МРТ сердца с контрастированием позволяет визуализировать тонкий предсердный миокард как у пациентов с впервые выявленной мерцательной аритмией, так и у лиц, перенесших интервенционные вмешательства.

В настоящее время есть все основания полагать, что МРТ-данные о структуре предсердий при фибрилляции предсердий в ближайшей перспективе обеспечат совершенствование тактики ведения таких пациентов.

Ключевые слова: фибрилляция предсердий, структурное ремоделирование, артериальная гипертензия, фиброз левого предсердия, магнитно-резонансная томография сердца с отсроченным контрастированием, моделирование сердца.

Ссылка для цитирования: Апарина О.П., Стукалова O.В., Терновой С.К. Магнитно-резонансная томография с отсроченным контрастированием при фибрилляции предсердий: первые достижения и перспективы. Медицинская визуализация. 2017; 21 (4): 47-57. DOI: 10.24835/1607-0763-2017-4-47-57.

$$
\star \star \star ~
$$

Current paper reviews new opportunities in none invasive evaluation of the left atrial fibrosis in atrial fibrillation using late gadolinium enhancement magnetic resonance imaging. The first use of this technology in clinical practice is discussed.

In a group of prospective studies it has been shown that structural atrial remodeling can influence the clinical course of atrial fibrillation and the efficiency of arrhythmia treatment. Several groups of national and European experts in morphology and MRI studies in a consensus suggest introduction of a new term fibrous atrial cardiomyopathy or fibrous car- 
diomyopathy of atrii, representing the structural and functional pathology of atrii, of clinical value in atrial fibrillation. These data make evidence for possibly wide noninvasive MRI studies of cardiovascular out-patients in order to make obvious the structure of atrial myocardium and atrial anatomy as a whole.

Progress in MRI technologies of heart studies provides the possibility of imaging of thin atrial myocardium both in patients with primarily documented atrial fibrillation and in persons after interventional treatment of the arrhythmia.

There are reasons to expect that LGE MRI data regarding atrial structure in atrial fibrillation are in a position to provide in a nearest future the improvement of management of such patients.

Key words: atrial fibrillation, hypertension, structural remodeling, left atrial fibrosis, late gadolinium enhancement magnetic resonance imaging, heart modeling.

Recommended citation: Aparina O.P., Stukalova O.V., Ternovoy S.K. Magnetic Resonance Imaging with Delayed Contrast Enhancement in Atrial Fibrillation: First Advances and Perspectives. Medical visualization. 2017; 21 (4): 47-57. DOI: 10.24835/1607-0763-2017-4-47-57.

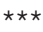

\section{Введение}

В современной диагностике болезней сердца широко используют неинвазивные исследования сердца и сосудов. Согласно отечественным и международным рекомендациям, при выборе тактики ведения кардиологических пациентов специалисты все чаще опираются на данные томографических исследований сердца [1]. В связи с необходимостью точной оценки анатомии камер сердца и внедрением технологий обработки и реконструкции изображений томографические методики совершенствуются в направлении увеличения разрешающей способности исследования [2].
Магнитно-резонансная томография (МРT) сердца с отсроченным контрастированием является "золотым стандартом" диагностики рубцового поражения миокарда левого желудочка ишемической и неишемической этиологии [3]. Высокую диагностическую ценность данный метод приобрел вследствие убедительных доказательств соответствия зон накопления гадолинийсодержащего контрастного препарата (отсроченного контрастирования через 15-20 мин после его внутривенного введения) на МР-томограммах зонам некроза, рубца и диффузного фиброза, подтвержденным патоморфологически [4].

В 1990-2000 гг. ряд патоморфологических и экспериментальных работ убедительно продемонстрировали наличие очагов воспаления и интерстициального фиброза в предсердном миокарде при такой распространенной сердечной аритмии, как фибрилляция предсердий $[5,6]$. Выявленные морфологические феномены наряду с истончением стенок предсердий и расширением объема его полости получили название "структурное ремоделирование предсердий” [7]. В ряде проспективных исследований было показано, что выраженность поражения предсердного миокарда может влиять на клиническое течение аритмии и результаты ее лечения [8]. В связи с этим ряд европейских экспертов в совместном консенсусе предлагают ввести термин "фиброзная кардиомиопатия предсердий”, отражающий структурнофункциональную патологию предсердий, имеющую клиническую значимость при фибрилляции предсердий [8, 9]. Эти данные обосновывают потребность в неинвазивном обследовании широкого круга кардиологических пациентов с целью оценки структуры предсердного миокарда.

Для корреспонденции*: Стукалова Ольга Владимировна - 121552 Москва, ул. 3-я Черепковская, 15а. Отдел томографии НИИ клинической кардиологии им. А.Л. Мясникова ФГБУ "Российский кардиологический научно-производственный комплекс" МЗ РФ. Тел.: +7-910 405 371. E-mail: olgastukalova@mail.ru

Апарина Ольга Петровна - канд. мед. наук, младший научный сотрудник отдела клиническойэлектрофизиологии и рентгенохирургических методов лечения нарушений ритма сердца НИИ клинической кардиологии им. А.Л. Мясникова ФГБУ "Российский кардиологический научно-производственный комплекс" МЗ РФ; Стукалова Ольга Владимировна - канд. мед. наук, старший научный сотрудник отдела томографии НИИ клинической кардиологии им. А.Л. Мясникова ФГБУ "Российский кардиологический научно-производственный комплекс" МЗ РФ; Терновой Сергей Константинович - академик РАН, доктор мед. наук, профессор, руководитель отдела томографии НИИ клинической кардиологии им. А.Л. Мясникова ФГБу "Российский кардиологический научно-производственный комплекс" МЗ РФ, заведующий кафедрой лучевой диагностики и лучевой терапии ФГАОУ ВО Первый Московский государственный медицинский университет им. И.М. Сеченова МЗ РФ (Сеченовский Университет).

Contact*: Olga V. Stukalova - 3-rd Cherepkovskaya, 15A, Moscow, Russia. Department of tomography, A.L. Mjasnikov Institute of the clinical cardiology of Russian Cardiologic research and production complex. Phone: +7-910 405 3711. E-mail: olgastukalova@mail.ru

Olga P. Aparina - cand. of med. sci., junior research fellow of the Department of clinical electrophysiology and radiosurgical treatment methods of heart arrhythmias of the A.L. Mjasnikov Institute of the clinical cardiology of Russian Cardiologic research and production complex, Moscow, Russia; Olga V. Stukalova - cand. of med. sci., senior research fellow of the Department of tomography of A.L. Mjasnikov Institute of the clinical cardiology of Russian Cardiologic research and production complex, Moscow, Russia; Sergey K. Ternovoy - Academician of the Russian Academy of Sciences, doct. of med. sci., professor, Chairman of the Department of tomography of A.L. Mjasnikov Institute of the clinical cardiology of Russian Cardiologic research and production complex; Chairman of the chair of radiology of I.M. Sechenov First Moscow state medical university of (Sechenov University). 

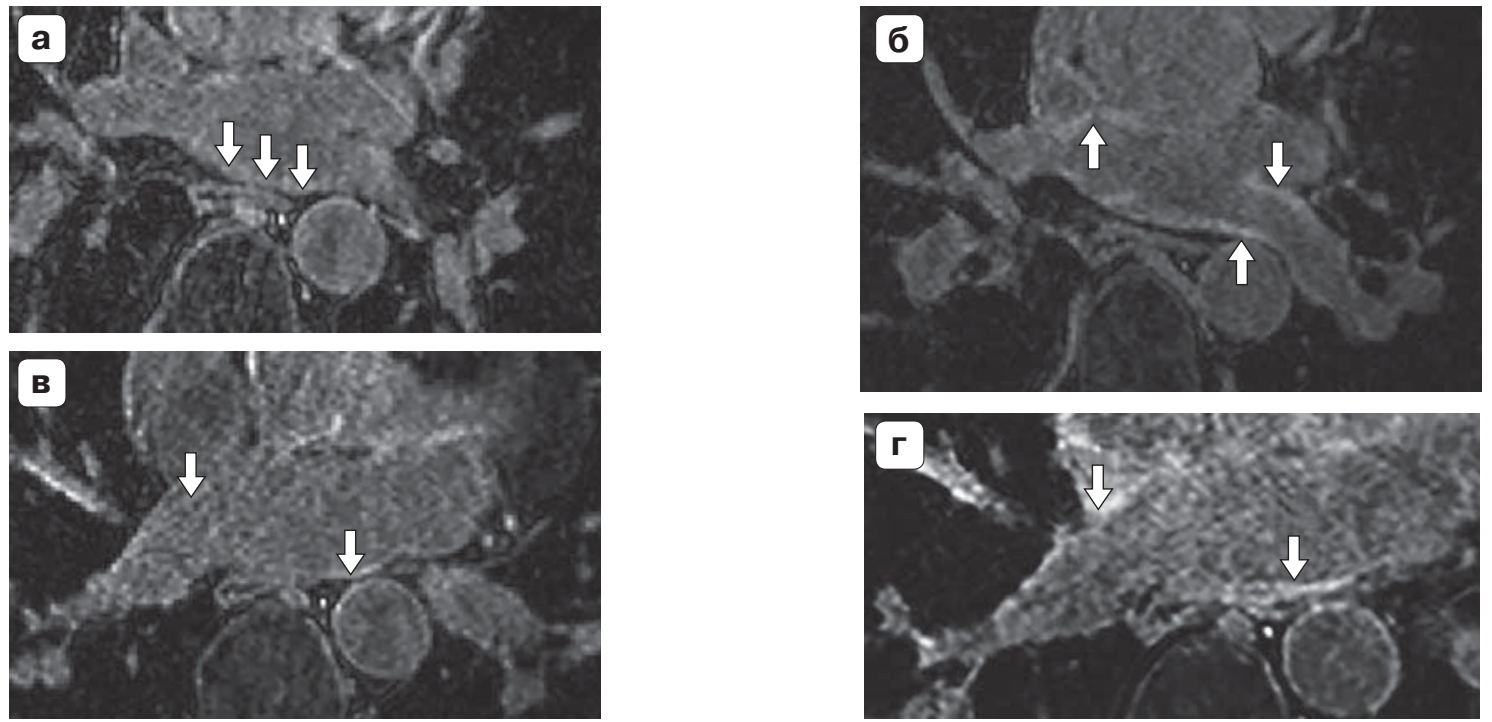

Рис. 1. МР-изображения миокарда левого предсердия высокого разрешения с отсроченным контрастированием. Область устьев правой и левой верхних легочных вен до (а) и после (б) абляции. Область устьев правой и левой нижних легочных вены до (в) и после (г) абляции (желтыми стрелками обозначен миокард левого предсердия, красными стрелками указаны появившиеся участки отсроченного контрастирования, соответствующие постабляционным повреждениям).

Визуализация миокарда предсердий стала возможна в последние годы благодаря разработке новых МР-импульсных последовательностей высокого разрешения. Усовершенствованные градиентные МР-последовательности обладают рядом свойств, позволяющих успешно получить изображения тонкого предсердного миокарда $[10,11]$. Этими свойствами являются высокое временно́е и пространственное разрешение, возможность коррекции артефактов движения предсердий в сердечном и дыхательном циклах и артефактов тока крови в легочных венах. Неинвазивность и удовлетворительная переносимость больными в совокупности с возможностью оценить структурные изменения во всем объеме предсердного миокарда придают методу МРТ с отсроченным контрастированием статус одной из наиболее перспективных методик оценки структурного ремоделирования предсердий.

Пилотное исследование в этом направлении было проведено группой исследователей под руководством D. Peters [12]. Данная работа продемонстрировала возможность визуализации предсердного миокарда у пациентов с фибрилляцией предсердий, которым проводилась катетерная абляция устьев легочных вен. При сравнении МР-изображений до и после абляции на последних в области устьев легочных вен были выявлены регионы, интенсивно накопившие контрастный препарат. Эти регионы топографически соответствовали местам нанесения абляционных воздей- ствий. Появление зон отсроченного контрастирования соответствовало накоплению контрастного препарата в зонах некроза и воспаления, которые явились следствием повреждения предсердного миокарда радиочастотной энергией (рис. 1).

Особенности формирования картины постабляционных повреждений и их ассоциации с гистологической структурой миокарда подробно описаны рядом авторов [13-15]. Было установлено, что повреждения миокарда предсердий в течение первых суток после абляции, отраженные на MP-томограммах зонами интенсивного отсроченного контрастирования, представлены очагами отека, воспаления и некроза. Часть из этих зон, по-видимому, за счет отека и воспаления, регрессирует [15], а часть трансформируется в рубцовую ткань, которую можно выявлять при помощи МРТ [16] и в более поздние сроки. По результатам исследований было предложено изучать постабляционные повреждения не в первые 24 4, а сформировавшиеся постабляционные рубцы через 3 мес после абляции. Именно с этими данными о рубцовой изоляции легочных вен проводится сопоставление клинической эффективности вмешательства в опубликованных исследованиях.

Таким образом, в настоящее время есть все основания заключить, что МРТ высокого разрешения с отсроченным контрастированием позволяет оценивать постабляционные рубцы после катетерной изоляции легочных вен. Имеются данные проспективных одноцентровых исследо- 
ваний, демонстрирующих, что большее количество изолированных легочных вен независимо ассоциировано с успешностью вмешательства [17]. В то же время взаимосвязи рубцовой изоляции легочной вены с клиническими и электрофизиологическими критериями успешности интервенционного лечения противоречивы и требуют дальнейшего изучения.

Необходимо отдельно подчеркнуть, что оценка постабляцинных повреждений предсердного миокарда на МР-изображениях высокого разрешения является задачей, решение которой с высокой воспроизводимостью и точностью может быть осуществлено опытными экспертами-радиологами $[11,18]$. Постабляционные рубцы, накопившие контрастный препарат, хорошо визуализируются на МР-изображениях, что позволяет в двух- и трехмерном режимах оценивать их форму (замкнутость) и топографию. Изучение структуры постабляционных рубцов, выполненное независимыми группами исследователей, легло в основу следующей существенно более сложной задачи оценки "естественно" сформировавшихся очагов фиброза в структурно измененных предсердиях при фибрилляции предсердий.

Следует отметить, что зоны отсроченного контрастирования в предсердном миокарде могут отражать не только зоны фиброза и некроза, но и воспаления и отека, то есть широкий спектр структурных изменений миокарда $[15,19]$. Однако участки накопления контрастного препарата в миокарде левого предсердия по данным МРТ в литературе называют условно зонами фиброза $[8,20]$, поэтому в настоящей статье мы также придерживались данной терминологии, которая уже стала общепринятой.

Известно, что даже здоровый предсердный миокард отличается большим содержанием соединительнотканных волокон по сравнению с миокардом желудочков [21]. Данная гистологическая особенность в совокупности с малой толщиной миокарда (1,5-3 мм) обусловливает малую контрастность между интенсивностью сигнала нормального предсердного миокарда и мелкоочагово-мозаичных или диффузных зон фиброза. Приведенные обстоятельства значительно затрудняют достоверное и воспроизводимое определение экспертом патологически измененного миокарда предсердий на МР-изображениях [11]. Кроме того, крайне ограничено количество работ, анализирующих соответствие данных МРТ и гистологических изменений миокарда предсердий. Только в двух исследованиях, включивших небольшое количество больных, перенесших кардиохирургические вмешательства, было продемонстрировано соот- ветствие зон накопления контрастного препарата миокардом левого предсердия интерстициальному фиброзу в биоптатах из этих зон [22, 23].

Одним из решений проблемы операторской погрешности при выявлении фиброза предсердий явилось применение объективных автоматических методик для выделения этих участков [11]. Преимуществами автоматического определения зон фиброза перед простой визуальной оценкой экспертом являются стандартизация методики и воспроизводимость результатов. Авторы настоящего исследования, являющиеся первой научной группой в России, успешно получившей МР-изображения предсердий высокого разрешения, на основании собственного опыта считают, что вклад в выявление фиброза предсердий как эксперта, так и автоматического алгоритма имеет равную значимость [24]. Во-первых, эксперт-радиолог определяет качество МР-изображений, степень выраженности на них артефактов, их общую пригодность для анализа. Во-вторых, работа эксперта-радиолога включает точное выделение контуров предсердного миокарда, что требует единого алгоритма [25]. В-третьих, важным аспектом является настройка оптимальной яркости и контрастности миокарда и крови. Без тщательного проведения этого подготовительного этапа обработки МР-изображений точное выявление зон фиброза в предсердном миокарде невозможно. Вместе с тем важно подчеркнуть, что в опубликованных исследованиях практически отсутствуют подробные описания этой части работы.

Первые попытки сегментирования зон фиброза зарубежными и отечественными исследователями продемонстрировали, что сегментирование предсердного фиброза при помощи стандартного программного обеспечения рабочих станций МР-томографов, рассчитанного для работы с миокардом левого желудочка, не обладает достаточной точностью $[10,11]$. Выявленное ограничение послужило предпосылкой для разработки независимыми группами ученых специализированных методик, адаптированных для исследования предсердного миокарда.

Bсе предложенные методики основаны на определении характеристик интенсивности сигнала миокарда предсердий на МР-изображениях. Зоны фиброза, наиболее выраженно накопившие контрастный препарат, выявляют как регионы, обладающие наиболее интенсивным сигналом. В предложенных методиках применяется оценка сигнала миокарда предсердия при помощи характеристической кривой (гистограммы интенсивности вокселей) или на основании сравнения с интенсивностью сигнала крови $[10,11,26,27]$. 

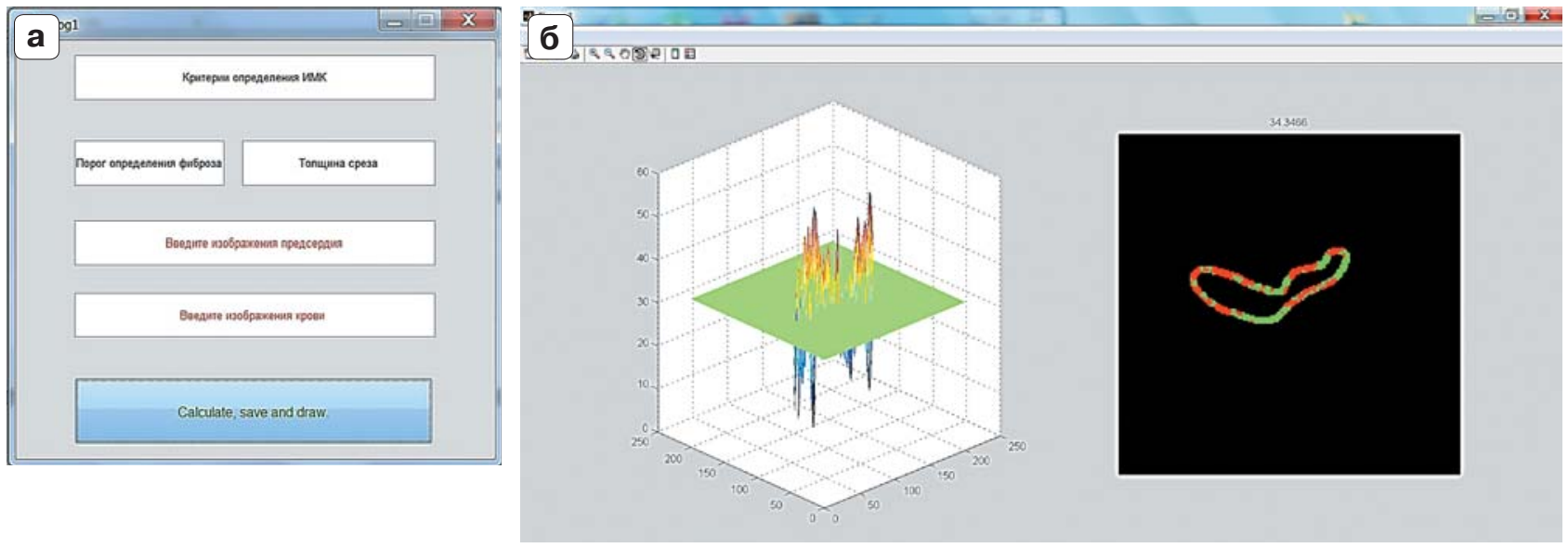

Рис. 2. Начальное окно ввода данных оригинальной программы LGE Heart Analyzer (a) и процесс выявления зон фиброза в программе LGE HEART Analyzer (б).

Среди ряда предложенных методов детекции предсердного фиброза наиболее оптимальный способ в настоящее время не определен. Имеются данные небольшого исследования, показавшего, что существующие современные методики имеют сопоставимые характеристики точности при поиске и количественной оценке зон фиброза в левом предсердии [11]. Кроме того, крайне ограничены данные о соответствии зон фиброза в левом предсердии по данным МРТ зонам фиброза, определенным патоморфологически.

Представленные выше предпосылки привели рентгенологов и кардиологов ФГБУ "РКНПК” в тесном сотрудничестве с кибернетиками из МГУ им. М.В. Ломоносова к разработке оригинальной методики выявления предсердного фиброза. В основе работы лежит обработка МР-изображений сердца с отсроченным контрастированием, которые были получены первоначально у здоровых добровольцев. На основании количественной оценки характеристик сигнала миокарда разработаны и внедрены показатели: индекс диффузного контрастирования (отношение средней интенсивности сигнала миокарда к средней интенсивности сигнала крови) и индекс максимального контрастирования (отношение максимальной интенсивности сигнала миокарда к средней интенсивности сигнала крови) [28].

Трудоемкость интегрального расчета данных параметров при необходимости их высокой точности явилась предпосылкой для создания нами специализированной программы LGE Heart Analyzer [29] (рис. 2).

Данная программа автоматически рассчитывает выраженность поражения миокарда предсердий, а также строит вращающиеся трехмер- ные модели левого предсердия с картированными зонами фиброза [29]. Количественная оценка разработанных показателей у здоровых лиц позволила установить критерии для выявления зон фиброза в миокарде предсердий и провести их количественную оценку [28].

Необходимо отметить, что в большинстве зарубежных методик в качестве референсных данных для детекции фиброза использовали их топографическое сопоставление с зонами снижения амплитуды электрического эндокардиального потенциала левого предсердия при его внутрисердечном картировании (лишь косвенно отражающими зоны миокардиального фиброза) [10, 26, 27]. Тем не менее эти данные еще раз подтвердили возможность детекции фиброза левого предсердия при помощи МРТ и легли в основу изучения связанных с ним клинических закономерностей течения фибрилляции.

В ряде работ было показано [10, 20, 30, 31], что у пациентов с фибрилляцией предсердий выраженность фиброза левого предсердия может колебаться от 0 до 70\%. Для оценки степени фиброзного поражения левого предсердия группа ученых под руководством N. Marrouche разработала шкалу, получившую название Utah [30]. B eе основу легли результаты МРТ с отсроченным контрастированием 333 больных фибрилляцией предсердий. В данной шкале был введен показатель объемной доли фиброза в миокарде левого предсердия, выраженной в процентах. Шкала Utah включает 4 степени: I - менее 5\% фиброза, II - 5-20\% фиброза, III - 20-35\% фиброза и IV более 35\% зон фиброза в предсердном миокарде. В исследовании О.В. Стукаловой и соавт. выраженность фиброзного поражения также варьи- 


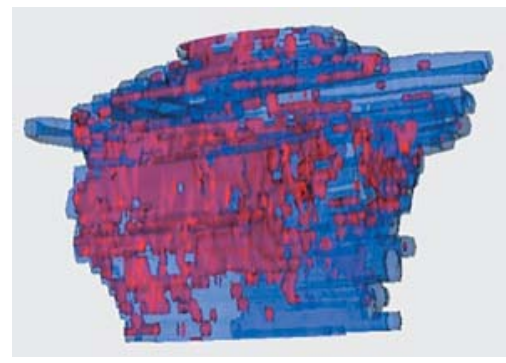

Рис. 3. Трехмерная модель левого предсердия с картированными зонами фиброза (красным) по задней стенке и области устьев легочных вен. Синим обозначен здоровый миокард.

ровала от 0 до 70\% от объема предсердного миокарда, составляя в среднем 9\% [31] (рис. 3).

В дальнейших зарубежных и отечественных работах было установлено, что большая выраженность фиброза левого предсердия ассоциирована с расширением полости левого предсердия и снижением его сократимости [24, 31-33]. Так, было показано, что у пациентов с фибрилляцией предсердий выраженность предсердного фиброза коррелировала с расширением полости левого предсердия и ухудшением его сократимости, что может отражать влияние замещения здорового миокарда фиброзной тканью на механическую функцию предсердий.

Эти данные могут объяснять результаты работы N. Akoum и соавт. [34], в которой было продемонстрировано, что у пациентов с более выраженным предсердным фиброзом в полостях предсердий чаще выявляли тромбы и отмечали более высокую градацию эффекта спонтанного контрастирования. Другими факторами, влияющими на выраженность предсердного фиброза у больных фибрилляцией предсердий, были артериальная гипертензия, сердечная недостаточность или гипертрофия левого желудочка [20, 31, 35-37].

Значительный интерес представляет вопрос о взаимосвязи между степенью фиброзного поражения предсердий и особенностями клинического течения фибрилляции предсердий. Следует подчеркнуть, что такие особенности клинического течения аритмии, как частота развития и длительность приступов, значительно варьируют не только у различных пациентов, но даже у одного пациента. K настоящему времени данные о связи выраженности фиброза в левом предсердии с клиническим течением фибрилляции предсердий остаются противоречивыми [10, 20, 24, 30, 31, 33]. Надежды клиницистов на возможность прогнозирования течения фибрилляции предсердий при помощи данных о выраженности предсердного фиброза в настоящее время не реализованы. Решение этой непростой задачи, по-видимому, требует тщательно спланированных проспективных наблюдений.

Несмотря на отсутствие прямых связей между фиброзным поражением предсердий и клинической картиной фибрилляции предсердий, изучение фиброза левого предсердия продолжает представлять актуальную задачу для клиницистов $[8,9]$. Это обосновано данными о влиянии выраженности и топографии фиброза на эффективность интервенционного лечения больных фибрилляцией предсердий. В настоящее время эффективность операции катетерной изоляции устьев легочных вен составляет около $50 \%[8,9]$. В связи с этим имеется серьезная потребность в поиске факторов, которые могут повысить эффективность вмешательства. Среди этих факторов немаловажная роль отводится структурному ремоделированию предсердий.

В пилотных работах R.S. Oakes и соавт. [10] и C. Mankopf и соавт. [30] было установлено, что у пациентов с выраженностью поражения предсердия, превышающей 35\% (Utah IV), абляция устьев легочных вен сопровождается почти $100 \%$ рецидивов аритмии, несмотря на хорошую технику выполнения вмешательства. У пациентов с меньшей выраженностью фиброза (0-35\%) катетерные вмешательства были существенно более эффективны с отсутствием рецидивов аритмии более чем в $50 \%$ случаев. Эти данные были подтверждены в дальнейшем в многоцентровом исследовании DECAAF, включившем 329 пациентов [38]. Полученные результаты позволили авторам предложить при выборе тактики ведения пациента с фибрилляцией предсердий руководствоваться данными МРТ предсердий в качестве независимого фактора. Пациентов с выраженным предсердным фиброзом (более 35\%) авторы предлагают лечить консервативно, так как польза от вмешательства не превышает потенциальные риски.

Другим направлением исследований в области МРТ предсердий является оценка специфичности предсердного фиброза для фибрилляции предсердий.

На основании обследования 23 здоровых добровольцев нами было показано, что у здоровых лиц со средним возрастом 50 лет может быть выявлен предсердный фиброз малой выраженности (всреднемнеболее 3,5\%) [39]. Примечательно, что немногочисленные зоны фиброза у здоровых лиц располагались преимущественно по задней стенке левого предсердия, примыкающей к кольцу митрального клапана, причем выраженность фиброза левого предсердия у здоровых лиц коррелировала с возрастом. Таким образом, выявление предсердного фиброза у лиц без сердечно- 
сосудистых заболеваний может отражать естественное старение сердца. В то же время у больных фибрилляцией предсердий, сопоставимых по возрасту со здоровыми лицами, фиброз левого предсердия был достоверно более выражен и расположен преимущественно в зонах устьев легочных вен и задней стенки левого предсердия. Топографические особенности зон фиброза левого предсердия у больных фибрилляцией предсердий могут быть объяснены результатами работ R.J. Hunter и соавт. [41] и E.S. Di Martino и соавт. [40]. Авторы установили, что растяжение левого предсердия при фибрилляции предсердий приводит $\mathrm{k}$ развитию значительного напряжения предсердной ткани именно в области устьев легочных вен и задней стенки, что может служить пусковым механизмом для развития структурных изменений в этой области предсердия.

В независимых исследованиях было показано, что предсердный фиброз может быть выявлен при артериальной гипертензии и хронической сердечной недостаточности даже в отсутствие приступов фибрилляции предсердий [31, 37, 39]. Таким образом, по-видимому, у здоровых лиц развитие предсердного фиброза может быть проявлением возрастных изменений сердца, тогда как у больных с сердечно-сосудистыми заболеваниями он может формироваться в условиях механической перегрузки предсердий при фибрилляции предсердий, систолической или диастолической дисфункции левого желудочка. Характеристические особенности топографии и выраженности предсердного фиброза при фибрилляции предсердий, несомненно, требуют дальнейшего изучения.

\section{Заключение}

Совершенствование технологии МРТ сердца с контрастированием позволяет визуализировать тонкий предсердный миокард. Крупные сердечнососудистые центры, имеющие в своем штате опытных радиологов, электрофизиологов, а также возможности привлечения специалистов по компьютерному анализу и моделированию, обладают возможностями для визуализации постабляционных рубцов и фиброза левого предсердия. В настоящее время имеются данные первых исследований, которые продемонстрировали, что данные о структуре предсердий при фибрилляции предсердий в перспективе могут помочь совершенствованию тактики ведения таких пациентов. В то же время ввиду новизны данной технологии остается нерешенным ряд вопросов по технологии получения и обработки МР-изображений высокого разрешения, изучению особенностей фиброза предсердий у больных с другими сер- дечно-сосудистыми заболеваниями. Есть все основания полагать, что результаты дальнейших исследований будут давать новые ответы на вопросы.

\section{Список литературы}

1. Akoum N., Daccarett M., McGann C., Segerson N., Vergara G., Kuppahally S., Badger T., Burgon N., Haslam T., Kholmovski E., Macleod R., Marrouche N. Atrial fibrosis helps select the appropriate patient and strategy in catheter ablation of atrial fibrillation: a DE-MRI guided approach. J. Cardiovasc. Electrophysiol. 2011; 22 (1): 16-22. DOI:10.1111/j.1540-8167.2010.01876.x.

2. Akcakaya M., Rayatzadeh H., Basha T.A., Hong S.N., Chan R.H., Kissinger K.V., Hauser T.H., Josephson M.E., Manning W.J., Nezafat R. Accelerated late gadolinium enhancement cardiac MR imaging with isotropic spatial resolution using compressed sensing: initial experience. Radiology. 2012; 264 (3): 691-699.

DOI:10.1148/radiol.12112489.

3. Flett A.S., Hasleton J., Cook C., Hausenloy D., Quarta G., Ariti C., Muthurangu V., Moon J.C. Evaluation of techniques for the quantification of myocardial scar of differing etiology using cardiac magnetic resonance. JACC Cardiovasc. Imaging. 2011; 4 (2): 150-156.

DOI:10.1016/j.jcmg.2010.11.015.

4. Kim R.J., Albert T.S., Wible J.H., Elliott M.D., Allen J.C., Lee J.C., Parker M., Napoli A., Judd R.M. Performance of delayed-enhancement magnetic resonance imaging with gadoversetamide contrast for the detection and assessment of myocardial infarction: an international, multicenter, double-blinded, randomized trial. Circulation. 2008; 117 (5): 629-637.

DOI:10.1161/CIRCULATIONAHA. 107.723262.

5. Morillo C.A., Klein G.J., Jones D.L., Guiraudon C.M. Chronic rapid atrial pacing. Structural, functional, and electrophysiological characteristics of a new model of sustained atrial fibrillation. Circulation. 1995; 91 (5): 1588-1595.

6. Frustaci A., Chimenti C., Bellocci F., Morgante E., Russo M.A., Maseri A. Histological substrate of atrial biopsies in patients with lone atrial fibrillation. Circulation. 1997; 96 (4): 1180-1184.

7. Aparina O.P., Chikhireva L.N., Mironova N.A., Mironova E.S., Bakalov S.A. [Role of atrial structural and functional changes in the development and progression of atrial fibrillation]. Ter. Arkh. 2014; 86 (1): 71-77.

8. Kottkamp H. Human atrial fibrillation substrate: towards a specific fibrotic atrial cardiomyopathy. Eur. Heart J. 2013; 34 (35): 2731-2738. DOI:10.1093/eurheartj/eht194.

9. Goette A., Kalman J.M., Aguinaga L., Akar J., Cabrera J.A., Chen S.A., Chugh S.S., Corradi D., A D.A., Dobrev D., Fenelon G., Gonzalez M., Hatem S.N., Helm R., Hindricks G., Ho S.Y., Hoit B., Jalife J., Kim Y.H., Lip G.Y., Ma C.S., Marcus G.M., Murray K., Nogami A., Sanders P., Uribe W., Van Wagoner D.R., Nattel S. EHRA/HRS/ APHRS/SOLAECE expert consensus on Atrial cardiomyopathies: Definition, characterisation, and clinical implication. J. Arrhythm. 2016; 32 (4): 247-278. DOI:10.1016/j.joa.2016.05.002.

10. Oakes R.S., Badger T.J., Kholmovski E.G., Akoum N., Burgon N.S., Fish E.N., Blauer J.J., Rao S.N., DiBella E.V., Segerson N.M., Daccarett M., Windfelder J., McGann C.J., 
Parker D., MacLeod R.S., Marrouche N.F. Detection and quantification of left atrial structural remodeling with delayed-enhancement magnetic resonance imaging in patients with atrial fibrillation. Circulation. 2009; 119 (13): 1758-1767. DOI: 10.1161/CIRCULATIONAHA.108.811877.

11. Karim R., Housden R.J., Balasubramaniam M., Chen Z., Perry D., Uddin A., Al-Beyatti Y., Palkhi E., Acheampong P., Obom S., Hennemuth A., Lu Y., Bai W., Shi W., Gao Y., Peitgen H.O., Radau P., Razavi R., Tannenbaum A., Rueckert D., Cates J., Schaeffter T., Peters D., MacLeod R., Rhode K. Evaluation of current algorithms for segmentation of scar tissue from late gadolinium enhancement cardiovascular magnetic resonance of the left atrium: an open-access grand challenge. J. Cardiovasc. Magn. Reson. 2013; 15: 105.

DOI: $10.1186 / 1532-429 X-15-105$.

12. Peters D.C., Wylie J.V., Hauser T.H., Kissinger K.V., Botnar R.M., Essebag V., Josephson M.E., Manning W.J. Detection of pulmonary vein and left atrial scar after catheter ablation with three-dimensional navigator-gated delayed enhancement MR imaging: initial experience. Radiology. 2007; 243 (3): 690-695.

DOI:10.1148/radiol.2433060417.

13. Badger T.J., Oakes R.S., Daccarett M., Burgon N.S., Akoum N., Fish E.N., Blauer J.J., Rao S.N., Adjei-Poku Y., Kholmovski E.G., Vijayakumar S., Di Bella E.V., MacLeod R.S., Marrouche N.F. Temporal left atrial lesion formation after ablation of atrial fibrillation. Heart Rhythm. 2009; 6 (2): 161-168. DOI:10.1016/j.hrthm.2008.10.042.

14. Taclas J.E., Nezafat R., Wylie J.V., Josephson M.E., Hsing J., Manning W.J., Peters D.C. Relationship between intended sites of RF ablation and post-procedural scar in $\mathrm{AF}$ patients, using late gadolinium enhancement cardiovascular magnetic resonance. Heart Rhythm. 2010; 7 (4): 489-496. DOI:10.1016/j.hrthm.2009.12.007.

15. Arujuna A., Karim R., Caulfield D., Knowles B., Rhode K., Schaeffter T., Kato B., Rinaldi C.A., Cooklin M., Razavi R., O'Neill M.D., Gill J. Acute pulmonary vein isolation is achieved by a combination of reversible and irreversible atrial injury after catheter ablation: evidence from magnetic resonance imaging. Circ. Arrhythm. Electrophysiol. 2012; 5 (4): 691-700. DOI:10.1161/CIRCEP.111.966523.

16. McGann C., Kholmovski E., Blauer J., Vijayakumar S., Haslam T., Cates J., DiBella E., Burgon N., Wilson B., Alexander A., Prastawa M., Daccarett M., Vergara G., Akoum N., Parker D., MacLeod R., Marrouche N. Dark regions of no-reflow on late gadolinium enhancement magnetic resonance imaging result in scar formation after atrial fibrillation ablation. J. Am. Coll. Cardiol. 2011; 58 (2): 177-185. DOI:10.1016/j.jacc.2011.04.008.

17. Peters D.C., Wylie J.V., Hauser T.H., Nezafat R., Han Y., Woo J.J., Taclas J., Kissinger K.V., Goddu B., Josephson M.E., Manning W.J. Recurrence of atrial fibrillation correlates with the extent of post-procedural late gadolinium enhancement: a pilot study. JACC Cardiovasc. Imaging. 2009; 2 (3): 308-316.

DOI:10.1016/j.jcmg.2008.10.016.

18. Karim R.A.A., Brazier A. Automatic Segmentation of Left Atrial Scar from Delayed-Enhancement Magnetic Resonance Imaging. In: D.N. Metaxas and L. Axel (eds). FIMH Berlin Heidelberg Springer-Verlag, 2011: 63-70.

19. Mahrholdt H., Wagner A., Judd R.M., Sechtem U., Kim R.J. Delayed enhancement cardiovascular magnetic resonance assessment of non-ischaemic cardiomyo- pathies. Eur. Heart J. 2005; 26 (15): 1461-1474. DOI:10.1093/eurheartj/ehi258.

20. Marrouche N.F., Wilber D., Hindricks G., Jais P., Akoum N., Marchlinski F., Kholmovski E., Burgon N., Hu N., Mont L., Deneke T., Duytschaever M., Neumann T., Mansour M., Mahnkopf C., Herweg B., Daoud E., Wissner E., Bansmann P., Brachmann J. Association of atrial tissue fibrosis identified by delayed enhancement MRI and atrial fibrillation catheter ablation: the DECAAF study. JAMA. 2014; 311 (5): 498-506. DOI:10.1001/jama.2014.3.

21. S. Kim Suvarna. General Considerations and Anatomy. In: S. Kim Suvarna. Cardiac Pathology. A guide to current practice. Springer, 2013.

22. McGann C.J. S.F., Patel A. Left atrial structural remodeling on LGE MRI correlates with histology. New York, 2012; 3 (59.13): E1236.

23. McGann C., Akoum N., Patel A., Kholmovski E., Revelo P., Damal K., Wilson B., Cates J., Harrison A., Ranjan R., Burgon N.S., Greene T., Kim D., Dibella E.V., Parker D., Macleod R.S., Marrouche N.F. Atrial fibrillation ablation outcome is predicted by left atrial remodeling on MRI. Circ. Arrhythm. Electrophysiol. 2014; 7 (1): 23-30. DOI:10.1161/CIRCEP.113.000689.

24. Апарина О.П., Стукалова О.В., Пархоменко Д.В., Миронова Н.А., Буторова Е.А., Болотова М.Н., Макеев М.И., Терновой С.К., Голицын С.П. Характеристика структуры миокарда левого предсердия у больных мерцательной аритмией и здоровых добровольцев по данным магнитно-резонансной томографии с отсроченным контрастированием. Вестник аритмологии. 2014; 77: 5-12.

25. Пат. RU 2576816 Российская Федерация, МПК А61B 5/055 (2006.01), А61K 49/06 (2006.01). Способ определения контуров миокарда левого предсердия на MPизображениях с использованием мультипланарных реконструкций/ Авторы: Стукалова О.В., Апарина О.П., Пархоменко Д.В., Миронова Н.А., Терновой С.К., Голицын С.П.заявитель и патентообладатель ФГБУ "РКНПК" МЗ РФ. № 2015107013/14(011264), заявл.02.03.2015, опубл. 10.03.2016.

26. Spragg D.D., Khurram I., Zimmerman S.L., Yarmohammadi H., Barcelon B., Needleman M., Edwards D., Marine J.E., Calkins H., Nazarian S. Initial experience with magnetic resonance imaging of atrial scar and coregistration with electroanatomic voltage mapping during atrial fibrillation: success and limitations. Heart Rhythm. 2012; 9(12):2003-2009, DOI:10.1016/j.hrthm.2012.08.039.

27. Malcolme-Lawes L.C., Juli C., Karim R., Bai W., Quest R., Lim P.B., Jamil-Copley S., Kojodjojo P., Ariff B., Davies D.W., Rueckert D., Francis D.P., Hunter R., Jones D., Boubertakh R., Petersen S.E., Schilling R., Kanagaratnam P., Peters N.S. Automated analysis of atrial late gadolinium enhancement imaging that correlates with endocardial voltage and clinical outcomes: a 2-center study. Heart Rhythm. 2013; 10 (8): 1184-1191.DOI:10.1016/j.hrthm.2013.04.030.

28. Пат. 2549825 Российская Федерация, МПК А61В 5/055. Способ оценки структурных изменений миокарда предсердий у больных с нарушениями ритма сердца / Авторы: Стукалова О.В., Апарина О.П., Пархоменко Д.В., Миронова Н.А., Терновой С.К., Голицын С.П. Заявитель и патентообладатель ФГБУ "РКНПК” МЗ РФ. №2014105347/14, заявл. 14.02.2014, опубл. 27.04.2015. Бюл. № 12, 8 с. 
29. Свидетельство о государственной регистрации программы для ЭВМ №2013661886. LGE Heart Analyzer. Заявители и правообладатели: Пархоменко Д.В., Апарина О.П., Стукалова О.В. Реестр программ для ЭВМ РФ 18.12.2013.

30. Mahnkopf C., Badger T.J., Burgon N.S., Daccarett M., Haslam T.S., Badger C.T., McGann C.J., Akoum N., Kholmovski E., Macleod R.S., Marrouche N.F. Evaluation of the left atrial substrate in patients with lone atrial fibrillation using delayed-enhanced MRI: implications for disease progression and response to catheter ablation. Heart Rhythm. 2010; 7 (10):1475-1481.

DOI:10.1016/j.hrthm.2010.06.030.

31. Стукалова О.В., Апарина О.П., Пархоменко Д.В., Терновой С.К. Оценка структурных изменений левого предсердия у больных мерцательной аритмией методом магнитно-резонансной томографии с отсроченным контрастированием. Российский электронный журнал лучевой диагностики. 2014; 4 (4): 8.

32. Kuppahally S.S., Akoum N., Burgon N.S., Badger T.J., Kholmovski E.G., Vijayakumar S., Rao S.N., Blauer J., Fish E.N., Dibella E.V., Macleod R.S., McGann C., Litwin S.E., Marrouche N.F. Left atrial strain and strain rate in patients with paroxysmal and persistent atrial fibrillation: relationship to left atrial structural remodeling detected by delayed-enhancement MRI. Circ. Cardiovasc. Imaging. 2010; 3 (3): 231-239.

DOI:10.1161/CIRCIMAGING.109.865683.

33. Стукалова О.В., Апарина О.П., Миронова Н.А., Голицын С.П. Фиброз миокарда левого предсердия по данным магнитно-резонансой томографии с отсроченным контрастированием у больных фибрилляцией предсердий. Альманах клинической медицины. 2015; 43: 9.

34. Akoum N., Fernandez G., Wilson B., McGann C., Kholmovski E., Marrouche N. Association of atrial fibrosis quantified using LGE-MRI with atrial appendage thrombus and spontaneous contrast on transesophageal echocardiography in patients with atrial fibrillation. J. Cardiovasc. Electrophysiol. 2013; 24 (10): 1104-1109. DOI: $10.1111 /$ jce. 12199.

35. Akkaya M., Higuchi K., Koopmann M., Damal K., Burgon N.S., Kholmovski E., McGann C., Marrouche N. Higher degree of left atrial structural remodeling in patients with atrial fibrillation and left ventricular systolic dysfunction. J. Cardiovasc. Electrophysiol. 2013; 24 (5): 485-491. DOI:10.1111/jce.12090.

36. Akkaya M., Marrouche N., Higuchi K., Koopmann M., Damal K., Kholmovski E., McGann C. The degree of left atrial structural remodeling impacts left ventricular ejection fraction in patients with atrial fibrillation. Turk. Kardiyol. Dern. Ars. 2014; 42 (1): 11-19. DOI: 10.5543/tkda.2014.20726.

37. Cochet H., Mouries A., Nivet H., Sacher F., Derval N., Denis A., Merle M., Relan J., Hocini M., Haissaguerre M., Laurent F., Montaudon M., Jais P. Age, atrial fibrillation, and structural heart disease are the main determinants of left atrial fibrosis detected by delayed-enhanced magnetic resonance imaging in a general cardiology population. J. Cardiovasc. Electrophysiol. 2015; 26 (5): 484-492. DOI:10.1111/jce.12651.

38. Akoum N., Wilber D., Hindricks G., Jais P., Cates J., Marchlinski F., Kholmovski E., Burgon N., Hu N., Mont L., Deneke T., Duytschaever M., Neumann T., Mansour M.,
Mahnkopf C., Hutchinson M., Herweg B., Daoud E., Wissner E., Brachmann J., Marrouche N.F. MRI Assessment of Ablation-Induced Scarring in Atrial Fibrillation: Analysis from the DECAAF Study. J. Cardiovasc. Electrophysiol. 2015; 26 (5): 473-480. DOI:10.1111/jce.12650.

39. Апарина О.П. Особенности структурного ремоделирования предсердий у пациентов с мерцательной аритмией: Автореф. дисс. канд. мед. наук. М. 2015. 22 с.

40. Di Martino E.S., Bellini C., Schwartzman D.S. In vivo porcine left atrial wall stress: Computational model. J. Biomech. 2011; 44 (15): 2589-2594.

DOI: 10.1016/j.jbiomech.2011.08.023.

41. Hunter R.J., Liu Y., Lu Y., Wang W., Schilling R.J. Left atrial wall stress distribution and its relationship to electrophysiologic remodeling in persistent atrial fibrillation. Circ. Arrhythm. Electrophysiol. 2012; 5 (2): 351-360. DOI:10.1161/CIRCEP.111.965541.

\section{References}

1. Akoum N., Daccarett M., McGann C., Segerson N., Vergara G., Kuppahally S., Badger T., Burgon N., Haslam T., Kholmovski E., Macleod R., Marrouche N. Atrial fibrosis helps select the appropriate patient and strategy in catheter ablation of atrial fibrillation: a DE-MRI guided approach. J. Cardiovasc. Electrophysiol. 2011; 22 (1): 16-22. DOI:10.1111/j.1540-8167.2010.01876.x.

2. Akcakaya M., Rayatzadeh H., Basha T.A., Hong S.N., Chan R.H., Kissinger K.V., Hauser T.H., Josephson M.E., Manning W.J., Nezafat R. Accelerated late gadolinium enhancement cardiac MR imaging with isotropic spatial resolution using compressed sensing: initial experience. Radiology. 2012; 264 (3): 691-699.

DOI:10.1148/radiol.12112489.

3. Flett A.S., Hasleton J., Cook C., Hausenloy D., Quarta G., Ariti C., Muthurangu V., Moon J.C. Evaluation of techniques for the quantification of myocardial scar of differing etiology using cardiac magnetic resonance. JACC Cardiovasc. Imaging. 2011; 4 (2): 150-156.

DOI:10.1016/j.jcmg.2010.11.015.

4. Kim R.J., Albert T.S., Wible J.H., Elliott M.D., Allen J.C., Lee J.C., Parker M., Napoli A., Judd R.M. Performance of delayed-enhancement magnetic resonance imaging with gadoversetamide contrast for the detection and assessment of myocardial infarction: an international, multicenter, double-blinded, randomized trial. Circulation. 2008; 117 (5): 629-637.

DOI:10.1161/CIRCULATIONAHA.107.723262.

5. Morillo C.A., Klein G.J., Jones D.L., Guiraudon C.M. Chronic rapid atrial pacing. Structural, functional, and electrophysiological characteristics of a new model of sustained atrial fibrillation. Circulation. 1995; 91 (5): 1588-1595.

6. Frustaci A., Chimenti C., Bellocci F., Morgante E., Russo M.A., Maseri A. Histological substrate of atrial biopsies in patients with lone atrial fibrillation. Circulation. 1997; 96 (4): 1180-1184.

7. Aparina O.P., Chikhireva L.N., Mironova N.A., Mironova E.S., Bakalov S.A. [Role of atrial structural and functional changes in the development and progression of atrial fibrillation]. Ter. Arkh. 2014; 86 (1): 71-77.

8. Kottkamp H. Human atrial fibrillation substrate: towards a specific fibrotic atrial cardiomyopathy. Eur. Heart J. 2013; 34 (35): 2731-2738. DOI:10.1093/eurheartj/eht194. 
9. Goette A., Kalman J.M., Aguinaga L., Akar J., Cabrera J.A., Chen S.A., Chugh S.S., Corradi D., A D.A., Dobrev D., Fenelon G., Gonzalez M., Hatem S.N., Helm R., Hindricks G., Ho S.Y., Hoit B., Jalife J., Kim Y.H., Lip G.Y., Ma C.S., Marcus G.M., Murray K., Nogami A., Sanders P., Uribe W., Van Wagoner D.R., Nattel S. EHRA/HRS/ APHRS/SOLAECE expert consensus on Atrial cardiomyopathies: Definition, characterisation, and clinical implication. J. Arrhythm. 2016; 32 (4): 247-278. DOI:10.1016/j.joa.2016.05.002.

10. Oakes R.S., Badger T.J., Kholmovski E.G., Akoum N., Burgon N.S., Fish E.N., Blauer J.J., Rao S.N., DiBella E.V., Segerson N.M., Daccarett M., Windfelder J., McGann C.J., Parker D., MacLeod R.S., Marrouche N.F. Detection and quantification of left atrial structural remodeling with delayed-enhancement magnetic resonance imaging in patients with atrial fibrillation. Circulation. 2009; 119 (13): 1758-1767. DOI: 10.1161/CIRCULATIONAHA.108.811877.

11. Karim R., Housden R.J., Balasubramaniam M., Chen Z., Perry D., Uddin A., Al-Beyatti Y., Palkhi E., Acheampong P., Obom S., Hennemuth A., Lu Y., Bai W., Shi W., Gao Y., Peitgen H.O., Radau P., Razavi R., Tannenbaum A., Rueckert D., Cates J., Schaeffter T., Peters D., MacLeod R., Rhode K. Evaluation of current algorithms for segmentation of scar tissue from late gadolinium enhancement cardiovascular magnetic resonance of the left atrium: an open-access grand challenge. J. Cardiovasc. Magn. Reson. 2013; 15: 105.

DOI: 10.1186/1532-429X-15-105.

12. Peters D.C., Wylie J.V., Hauser T.H., Kissinger K.V., Botnar R.M., Essebag V., Josephson M.E., Manning W.J. Detection of pulmonary vein and left atrial scar after catheter ablation with three-dimensional navigator-gated delayed enhancement MR imaging: initial experience. Radiology. 2007; 243 (3): 690-695.

DOI:10.1148/radiol.2433060417.

13. Badger T.J., Oakes R.S., Daccarett M., Burgon N.S., Akoum N., Fish E.N., Blauer J.J., Rao S.N., Adjei-Poku Y., Kholmovski E.G., Vijayakumar S., Di Bella E.V., MacLeod R.S., Marrouche N.F. Temporal left atrial lesion formation after ablation of atrial fibrillation. Heart Rhythm. 2009; 6 (2): 161-168. DOI:10.1016/j.hrthm.2008.10.042.

14. Taclas J.E., Nezafat R., Wylie J.V., Josephson M.E., Hsing J., Manning W.J., Peters D.C. Relationship between intended sites of RF ablation and postprocedural scar in AF patients, using late gadolinium enhancement cardiovascular magnetic resonance. Heart Rhythm. 2010; 7 (4): 489-496.

DOI:10.1016/j.hrthm.2009.12.007.

15. Arujuna A., Karim R., Caulfield D., Knowles B., Rhode K., Schaeffter T., Kato B., Rinaldi C.A., Cooklin M., Razavi R., O'Neill M.D., Gill J. Acute pulmonary vein isolation is achieved by a combination of reversible and irreversible atrial injury after catheter ablation: evidence from magnetic resonance imaging. Circ. Arrhythm. Electrophysiol. 2012; 5 (4): 691-700. DOI:10.1161/CIRCEP.111.966523.

16. McGann C., Kholmovski E., Blauer J., Vijayakumar S., Haslam T., Cates J., DiBella E., Burgon N., Wilson B., Alexander A., Prastawa M., Daccarett M., Vergara G., Akoum N., Parker D., MacLeod R., Marrouche N. Dark regions of no-reflow on late gadolinium enhancement magnetic resonance imaging result in scar formation after atrial fibrillation ablation. J. Am. Coll. Cardiol. 2011; 58 (2): 177-185. DOI:10.1016/j.jacc.2011.04.008.
17. Peters D.C., Wylie J.V., Hauser T.H., Nezafat R., Han Y., Woo J.J., Taclas J., Kissinger K.V., Goddu B., Josephson M.E., Manning W.J. Recurrence of atrial fibrillation correlates with the extent of post-procedural late gadolinium enhancement: a pilot study. JACC Cardiovasc. Imaging. 2009; 2 (3): 308-316.

DOI:10.1016/j.jcmg.2008.10.016.

18. Karim R.A.A., Brazier A. Automatic Segmentation of Left Atrial Scar from Delayed-Enhancement Magnetic Resonance Imaging. In: D.N. Metaxas and L. Axel (eds). FIMH Berlin Heidelberg Springer-Verlag, 2011: 63-70.

19. Mahrholdt H., Wagner A., Judd R.M., Sechtem U., Kim R.J. Delayed enhancement cardiovascular magnetic resonance assessment of non-ischaemic cardiomyopathies. Eur. Heart J. 2005; 26 (15): 1461-1474. DOI:10.1093/eurheartj/ehi258.

20. Marrouche N.F., Wilber D., Hindricks G., Jais P., Akoum N., Marchlinski F., Kholmovski E., Burgon N., Hu N., Mont L., Deneke T., Duytschaever M., Neumann T., Mansour M., Mahnkopf C., Herweg B., Daoud E., Wissner E., Bansmann P., Brachmann J. Association of atrial tissue fibrosis identified by delayed enhancement MRI and atrial fibrillation catheter ablation: the DECAAF study. JAMA. 2014; 311 (5): 498-506. DOI:10.1001/jama.2014.3.

21. S. Kim Suvarna. General Considerations and Anatomy. In: S. Kim Suvarna. Cardiac Pathology. A guide to current practice. Springer 2013 -.

22. McGann C.J. S.F., Patel A. Left atrial structural remodeling on LGE MRI correlates with histology. New York, 2012; 3 (59.13): E1236.

23. McGann C., Akoum N., Patel A., Kholmovski E., Revelo P., Damal K., Wilson B., Cates J., Harrison A., Ranjan R., Burgon N.S., Greene T., Kim D., Dibella E.V., Parker D., Macleod R.S., Marrouche N.F. Atrial fibrillation ablation outcome is predicted by left atrial remodeling on MRI. Circ. Arrhythm. Electrophysiol. 2014; 7 (1): 23-30. DOI: $10.1161 /$ CIRCEP.113.000689.

24. Aparina O.P., Stukalova O.V., Parkhomenko D.V., Mironova N.A., Golitsyn S.P. Characteristics of left atrial myocardium structure in atrial fibrillation and healthy volunteers using late gadolinium enhancement magnrtic resonance imaging. Vestnik arithmologii. 2014; 77 :5-12. (In Russian)

25. Patent RU 2576816. Russian Federation, MПK A61B 5/055 (2006.01), A61K 49/06 (2006.01) The method of definition of the borders of left atrial myocardium on MRimages with late gadolinium enhancement using multiplanar reconstructions. Authors: Stukalova O.V., Aparina O.P., Parkhomenko D.V., Mironova N.A., Ternovoy S.K., Golitsyn S.P. Owner FSBI RCRPC MH of RF № 2015107013/14(011264) stated.02.03.2015, published. 10.03.2016. (In Russian)

26. Spragg D.D., Khurram I., Zimmerman S.L., Yarmohammadi H., Barcelon B., Needleman M., Edwards D., Marine J.E., Calkins H., Nazarian S. Initial experience with magnetic resonance imaging of atrial scar and coregistration with electroanatomic voltage mapping during atrial fibrillation: success and limitations. Heart Rhythm. 2012;9(12):2003-2009, DOI:10.1016/j.hrthm.2012.08.039.

27. Malcolme-Lawes L.C., Juli C., Karim R., Bai W., Quest R., Lim P.B., Jamil-Copley S., Kojodjojo P., Ariff B., Davies D.W., Rueckert D., Francis D.P., Hunter R., Jones D., Boubertakh R., Petersen S.E., Schilling R., Kanagaratnam P., Peters N.S. Automated analysis of 
atrial late gadolinium enhancement imaging that correlates with endocardial voltage and clinical outcomes: a 2-center study. Heart Rhythm. 2013; 10 (8): 1184-1191.DOI:10.1016/j.hrthm.2013.04.030.

28. Patent RU 2549825. Russian Federation, МПК А61B 5/055 The method of evaluation of sttructural changes in atrial myocardium in patients with arrhythmias. Autors: Stukalova O.V., Aparina O.P., Parkhomenko D.V., Mironova N.A., Ternovoy S.K., Golitsyn S.P. Owner FSBI RCRPC MH of RF № 2015107013/14(011264) stated. 14.02.2014, published. 27.04.2015 Bul. № 12-8 (Пат. 2549825 Российская федерация, МПК А61B 5/055. (In Russian)

29. The certificate of state registration of programm for computer. LGE Heart Analyzer №2013661886. Copyright by Parkhomenko D.V., Aparina O.P., Stukalova O.V. Registry of computing programs of RF 18.12.2013. (In Russian)

30. Mahnkopf C., Badger T.J., Burgon N.S., Daccarett M., Haslam T.S., Badger C.T., McGann C.J., Akoum N., Kholmovski E., Macleod R.S., Marrouche N.F. Evaluation of the left atrial substrate in patients with lone atrial fibrillation using delayed-enhanced MRI: implications for disease progression and response to catheter ablation. Heart Rhythm. 2010; 7 (10):1475-1481. DOI:10.1016/j.hrthm.2010.06.030.

31. Stukalova O.V., Aparina O.P., Parkhomenko D.V., Ternovoy S.K. Evaluation of left atrial structiral changes in patients with atrial fibrillation using late gadolinium enhancement magnetic resonance imaging. REJR. 2014; 4 (4): 8. (In Russian)

32. Kuppahally S.S., Akoum N., Burgon N.S., Badger T.J., Kholmovski E.G., Vijayakumar S., Rao S.N., Blauer J., Fish E.N., Dibella E.V., Macleod R.S., McGann C., Litwin S.E., Marrouche N.F. Left atrial strain and strain rate in patients with paroxysmal and persistent atrial fibrillation: relationship to left atrial structural remodeling detected by delayed-enhancement MRI. Circ. Cardiovasc. Imaging. 2010; 3 (3): 231-239.

DOI:10.1161/CIRCIMAGING.109.865683.

33. Stukalova O.V., Aparina O.P., Mironova N.A., Golitsy S.P. Left atrial fibrosis in patients with atrial fibrillation according to magnetic resonance imaging with late gadolinium enhancement. Almanac of Clinical Medicine. 2015;(43): 29-37. (In Russian)

34. Akoum N., Fernandez G., Wilson B., McGann C., Kholmovski E., Marrouche N. Association of atrial fibrosis quantified using LGE-MRI with atrial appendage thrombus and spontaneous contrast on transesophageal echocardiography in patients with atrial fibrillation. J. Cardiovasc. Electrophysiol. 2013; 24 (10): 1104-1109. DOI: 10.1111/jce.12199.

35. Akkaya M., Higuchi K., Koopmann M., Damal K., Burgon N.S., Kholmovski E., McGann C., Marrouche N. Higher degree of left atrial structural remodeling in patients with atrial fibrillation and left ventricular systolic dysfunction. J. Cardiovasc. Electrophysiol. 2013; 24 (5): 485-491. DOI:10.1111/jce.12090.

36. Akkaya M., Marrouche N., Higuchi K., Koopmann M., Damal K., Kholmovski E., McGann C. The degree of left atrial structural remodeling impacts left ventricular ejection fraction in patients with atrial fibrillation. Turk. Kardiyol. Dern. Ars. 2014; 42 (1): 11-19. DOI: 10.5543/tkda.2014.20726.

37. Cochet H., Mouries A., Nivet H., Sacher F., Derval N., Denis A., Merle M., Relan J., Hocini M., Haissaguerre M., Laurent F., Montaudon M., Jais P. Age, atrial fibrillation, and structural heart disease are the main determinants of left atrial fibrosis detected by delayed-enhanced magnetic resonance imaging in a general cardiology population. J. Cardiovasc. Electrophysiol. 2015; 26 (5): 484-492. DOI:10.1111/jce.12651.

38. Akoum N., Wilber D., Hindricks G., Jais P., Cates J., Marchlinski F., Kholmovski E., Burgon N., Hu N., Mont L., Deneke T., Duytschaever M., Neumann T., Mansour M., Mahnkopf C., Hutchinson M., Herweg B., Daoud E., Wissner E., Brachmann J., Marrouche N.F. MRI Assessment of Ablation-Induced Scarring in Atrial Fibrillation: Analysis from the DECAAF Study. J. Cardiovasc. Electrophysiol. 2015; 26 (5): 473-480. DOI:10.1111/jce. 12650.

39. Aparina O.P.Features of structural remodelling of atrii in patients with atrial fibrillation. Abstract of Diss. Cand.Sci. Med. Moscow. 2015. 22 p.

40. Di Martino E.S., Bellini C., Schwartzman D.S. In vivo porcine left atrial wall stress: Computational model. J. Biomech. 2011; 44 (15): 2589-2594.

DOI: 10.1016/j.jbiomech.2011.08.023.

41. Hunter R.J., Liu Y., Lu Y., Wang W., Schilling R.J. Left atrial wall stress distribution and its relationship to electrophysiologic remodeling in persistent atrial fibrillation. Circ. Arrhythm. Electrophysiol. 2012; 5 (2): 351-360. DOI:10.1161/CIRCEP.111.965541.
Поступила в редакцию 7.06.2017. Принята к печати 30.06.2017.
Received on 7.06.2017.

Accepted for publication on 30.06.2017. 